\title{
Using Multidetector Row Computed Tomography to Evaluate Baggy Eyelid
}

\author{
Itsuko Okuda $\cdot$ Masahiro Irimoto $\cdot$ Yasuo Nakajima • \\ Shigemi Sakai $\cdot$ Kazuaki Hirata $\cdot$ Yukio Shirakabe
}

Received: 15 July 2011/ Accepted: 23 September 2011/Published online: 25 October 2011

(C) The Author(s) 2011. This article is published with open access at Springerlink.com

\begin{abstract}
Background Baggy eyelids, formed by intraorbital fat herniation in the lower eyelids, are a sign of aging observed in the midface. This study aimed to identify the cause of baggy eyelids by evaluating the relationship between orbicularis oculi muscle thickness, orbital fat prolapse length, and age using multidetector row computed tomography (MDCT).

Methods The 34 patients in the study ranged in age from 20 to 79 years. The patients were divided into three age groups: 20-29 years, 30-54 years, and 55-79 years.
\end{abstract}

\section{Okuda $(\square)$}

Department of Diagnostic Radiology, International University of Health and Welfare, Mita Hospital, 1-4-3, Mita Minato-ku, Tokyo 108-8329, Japan

e-mail: okudai-tora@umin.ac.jp

I. Okuda · Y. Nakajima

Department of Radiology, St. Marianna University School of Medicine, 2-16-1, Sugao Miyamae-Ku, Kawasaki City, Kanagawa 216-8511, Japan

\section{Irimoto}

Irimoto Medical, 1-12-10-203, Koishikawa, Bunkyo-ku,

Tokyo 112-0002, Japan

\section{S. Sakai}

Department of Plastic Surgery, International University of Health and Welfare, Mita Hospital, 1-4-3, Mita Minato-ku, Tokyo 108-8329, Japan

\section{K. Hirata}

Department of Anatomy, St. Marianna University School of Medicine, 2-16-1, Sugao Miyamae-Ku, Kawasaki City, Kanagawa 216-8511, Japan

Y. Shirakabe

Department of Aesthetic Plastic Surgery, Sapho Clinic,

5-17-16, Roppongi, Minato-ku, Tokyo 106-0032, Japan
Orbicularis oculi muscle thickness and orbital fat prolapse length were measured using reconstructed computed tomography (CT) images.

Results The orbicularis oculi muscle was found to be significantly thinner in the oldest group. Likewise, orbital fat prolapse was found to be significantly more prominent in the oldest group. A strong and significant negative correlation was found between orbicularis oculi muscle thickness and orbital fat prolapse length $(r=-0.78$; $P<0.001)$ and between orbicularis oculi muscle thickness and age $(r=-0.77 ; P<0.001)$. A strong and significant positive correlation was found between orbital fat prolapse length and age $(r=0.78 ; P<0.001)$.

Conclusions The use of MDCT provides insight into the detailed changes associated with aging that take place within the lower eyelids. This study objectively demonstrated that the major factors associated with baggy eyelids include orbicularis oculi muscle thickness, orbital fat prolapse length, and age. Decreased orbicularis oculi muscle thickness leads to the orbital fat prolapse.

Keywords Aging - Baggy eyelid - Herniation - Lower eyelid · Multidetector row computed tomography ·

Orbicularis oculi muscle $\cdot$ Orbital fat

Signs of aging on the face are caused by changes in soft tissues (i.e., muscles and fat) and in the skin itself, resulting in wrinkles and sagging [1-3]. Baggy eyelids, a sign of aging observed in the midface, are formed by intraorbital fat herniation in the lower eyelids. Common complaints may include lower eyelid bags, crescents under the eyes, and wrinkles around the eyes.

Goldberg et al. [4] reported that baggy eyelids are caused by a combination of several factors including 
orbital fat prolapse, eyelid fluid, tear trough depression, loss of skin elasticity, orbicularis prominence, and a triangular malar mound. Furthermore, Furnas [5] reported that baggy eyelids are attributable to laxity in the orbicularis oculi muscle. Using computed tomography (CT), Chen et al. [6] described the relationship between intraorbital fat herniation or orbital fat prolapse and age. To identify the cause of baggy eyelids by diagnostic imaging, we studied the relationship between orbicularis oculi muscle thickness, orbital fat prolapse length, and age.

\section{Materials and Methods}

\section{Population Selection}

Approval was obtained from our institutional review board to use the data from our previous multidetector row CT (MDCT) studies and facial imaging anatomy work based on three-dimensional (3D) CT. Patient anonymity was preserved, and the need for informed consent was waived due to the retrospective nature of the study.

From November 2010 through February 2011, the medical records of adult patients who had participated in a previous research study at our institution were reviewed. In that study, the sample population was composed of patients referred for a head and neck CT scan for various indications. Patients without lesions that would influence the superficial facial structure (e.g., nasal sinusitis evaluation and negative studies) were enrolled in the study. A total of 34 patients (25 men and 9 women) ranging in age from 20 to 79 years (mean, 44.9 years; range, 17.75 years) comprised the study group. The subjects were divided into three age groups: 20 to 29 years (10 patients), 30 to 54 years (11 patients), and 55 to 79 years (13 patients).

\section{CT Scan Technique}

Whole face scans were obtained using a 64-MDCT scanner (Aquilion 64; Toshiba Medical Systems, Tochigi, Japan). The scans were performed at a tube voltage of $120 \mathrm{kVp}$, a tube current of 130 to $220 \mathrm{~mA}$, a gantry rotation speed of $0.5 \mathrm{~s}$, a thickening of $1 \mathrm{~mm}$, a table speed of $30 \mathrm{~mm} / \mathrm{s}$, a collimation of $1 \mathrm{~mm}$, and a helical pitch of 15 . All imaging row data were transferred to a Centricity RA1000 workstation (GE Healthcare UK, Ltd, Buckinghamshire, UK), whereby 3D CT images, including reconstituted CT images, were created.

\section{Postprocessing Technique}

The workstation provided a menu of options composed of various preset reconstruction algorithms, including dedicated midface scan algorithms, which were used to reconstruct 3D CT images of the midface. Volume-rendering, based on an edge detection image processing system, was used in these $3 \mathrm{D}$ reconstructions.

\section{Baggy Eyelid Measurements}

The relationship between orbicularis oculi thickness and orbital fat prolapse was demonstrated by measurements using reconstructed images from MDCT data. Sagittal reconstructed CT images were created vertical to the orbit. For measuring orbital fat prolapse, the reference line was drawn from the anterior edge of the orbital roof to the anterior edge of the orbital floor. Orbital fat prolapse length was defined as the distance between the posterior margin of the orbicularis oculi muscle and the reference line. Orbicularis oculi muscle thickness was defined as the distance between the anterior margin and the posterior margin of the orbicularis oculi muscle.

Orbicularis oculi muscle thickness and orbital fat prolapse length were measured on data images using the digital caliper available on the workstation. The measurements were performed cooperatively by two radiologists, one with 18 years of experience (I.O.) and one with 30 years of experience (M.I.).

\section{Statistical Analysis}

Measured values of orbicularis oculi muscle thickness and orbital fat prolapse length were presented as mean \pm standard deviation and range. Mean values between the three age groups were compared using an independentsample $t$ test. A correlation analysis was performed to determine the relationship between orbicularis oculi muscle thickness and orbital fat prolapse length. Likewise, a correlation analysis was performed to determine the relationship between orbital fat prolapse length and age. Finally, a correlation analysis was performed to determine the relationship between orbicularis oculi muscle thickness and age. Statistical calculations were performed using the StatMate III statistical software package for Windows (ATMS, Tokyo, Japan). All $p$ values less than 0.05 were considered statistically significant.

\section{Results}

Measurement of Orbicularis Oculi Muscle Thickness and Orbital Fat Prolapse Length

The overall quality of the 3D CT images was adequate and did not hamper measurement of the baggy eyelids. The mean and standard deviation values, calculated using the 
measurements (Fig. 1) made by two observers, are shown in Table 1. The mean orbicularis oculi muscle thickness value was $2.2 \pm 0.57 \mathrm{~mm}$, and the mean orbital fat prolapse length was $3.7 \pm 2.03 \mathrm{~mm}$.

The orbicularis oculi muscle thickness was significantly thinner statistically in the 30- to 54-year-old group $(2.2 \pm 0.39 \mathrm{~mm})$ than in the 20 - to 29 -year-old group $(2.7 \pm 0.53 \mathrm{~mm})(P=0.018)$. The orbicularis oculi muscle thickness in the 55- to 79-year-old group $(1.7 \pm$ $0.35 \mathrm{~mm}$ ) was significantly thinner than in the $30-$ to 54-year-old group $(P<0.001)$.

The orbital fat prolapse length in the 30- to 54-year-old group $(3.3 \pm 1.56 \mathrm{~mm})$ was significantly more prominent than in the 20 - to 29 -year-old group $(1.6 \pm 1.45 \mathrm{~mm})$ $(P=0.02)$, and it was significantly more prominent in the 55 - to 79-year-old group $(5.7 \pm 1.46 \mathrm{~mm})$ than in the 30- to 54-year-old group $(P<0.001)$.

Analysis of the Relationship Between Orbicularis Oculi Muscle Thickness, Orbital Fat Prolapse Length, and Age

Our analysis found a strong and significant negative correlation between orbicularis oculi muscle thickness and orbital fat prolapse length (Fig. 2; $r=-0.78 ; P<0.001$ ). Specifically, as the orbicularis oculi muscle thickness decreased, the orbital fat prolapse length increased.

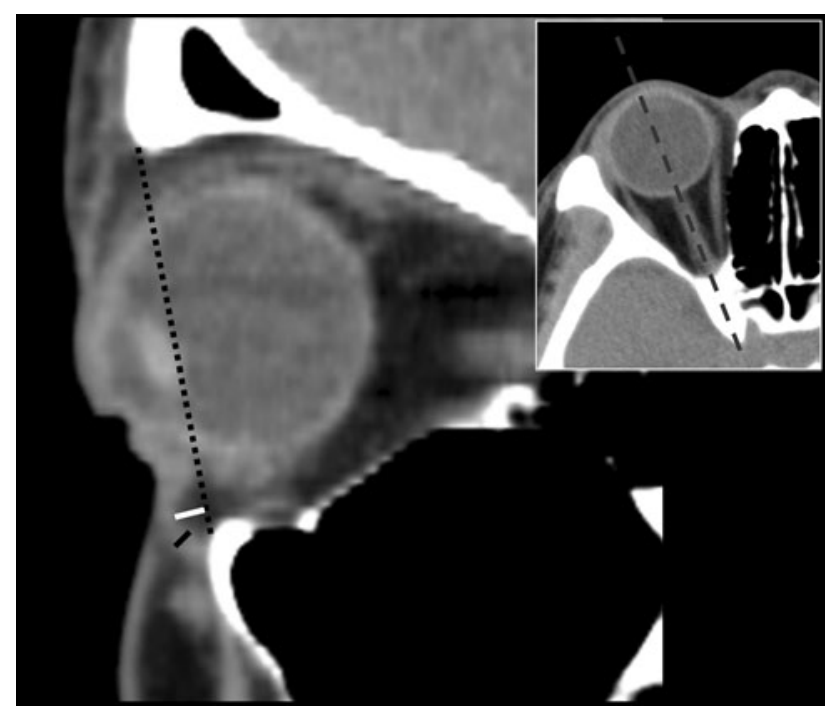

Fig. 1 Baggy eyelid measurements. Sagittal reconstructed images created vertical to the orbit (white box) were used for measurements of orbicularis oculi thickness and orbital fat prolapse. A line (dotted) running from the anterior edge of the orbital roof to the anterior edge of the orbital floor was used as a reference for measuring orbital fat prolapse length. White line: Orbital fat prolapse length, indicating the measurement made from the posterior margin of the orbicularis oculi muscle to the reference line. Black line: Orbicularis oculi muscle thickness measurement
Table 1 Measurements of orbicularis oculi muscle thickness and orbital fat prolapse length for the three age groups

\begin{tabular}{llll}
\hline $\begin{array}{l}\text { Age group } \\
\text { years) }\end{array}$ & $n$ & $\begin{array}{l}\text { Orbicularis oculi muscle } \\
\text { thickness }(\mathrm{mm})\end{array}$ & $\begin{array}{l}\text { Orbital fat prolapse } \\
\text { length }(\mathrm{mm})\end{array}$ \\
\hline $20-29$ & 10 & $2.7 \pm 0.53$ & $1.6 \pm 1.45$ \\
$30-54$ & 11 & $2.2 \pm 0.39$ & $3.3 \pm 1.56$ \\
$55-79$ & 13 & $1.7 \pm 0.35$ & $5.7 \pm 1.46$ \\
Total & 34 & $2.2 \pm 0.57$ & $3.7 \pm 2.03$ \\
\hline
\end{tabular}

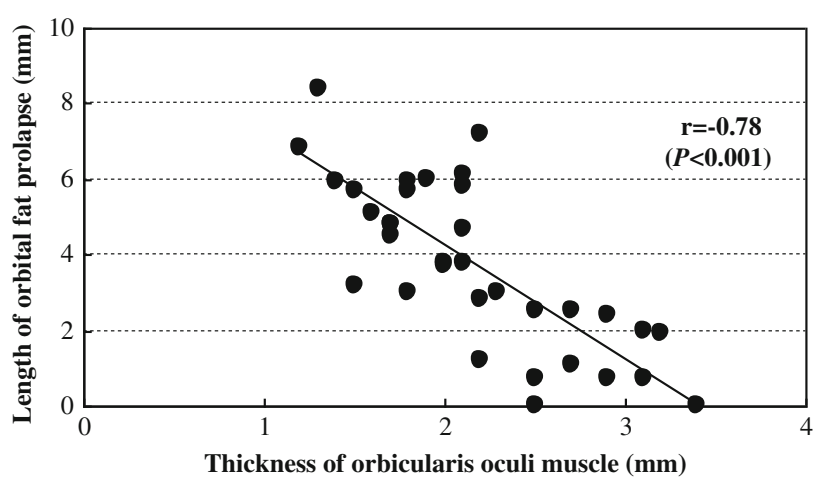

Fig. 2 Correlation between orbicularis oculi muscle thickness and orbital fat prolapse length. A strong and significant negative correlation was found between orbicularis oculi muscle thickness and orbital fat prolapse length $(r=-0.78 ; P<0.001)$. Specifically, as the orbicularis oculi muscle becomes thinner, the orbital fat prolapse becomes more prominent

Furthermore, with age, the orbicularis oculi muscle became thinner, and the orbital fat prolapse became more prominent. A strong and significant negative correlation was found between orbicularis oculi muscle thickness and age (Fig. 3; $r=-0.77 ; P<0.001$ ), whereas a strong and significant positive correlation was found between orbital fat prolapse length and age (Fig. 4; $r=0.78 ; P<0.001$ ). With younger age, the orbicularis oculi muscle was thicker and the orbital fat prolapse was milder (Fig. 5), whereas older age was found to correlate with decreased orbicularis oculi thickness and increased orbital fat prolapse (Fig. 6).

\section{Discussion}

An understanding of detailed facial anatomy is necessary for aesthetic plastic surgery procedures. Likewise, understanding the anatomy of the lower eyelid, including the effect of aging, assists in blepharoplasty. An increase in the use of MDCT and advancements in image analysis technology have made it feasible to create 3D CT images from routine images. Three-dimensional CT and reconstructed CT images provide detailed anatomic information [6-8].

Multiple factors have been implicated in the formation of baggy eyelids including orbital fat prolapse, eyelid fluid, 


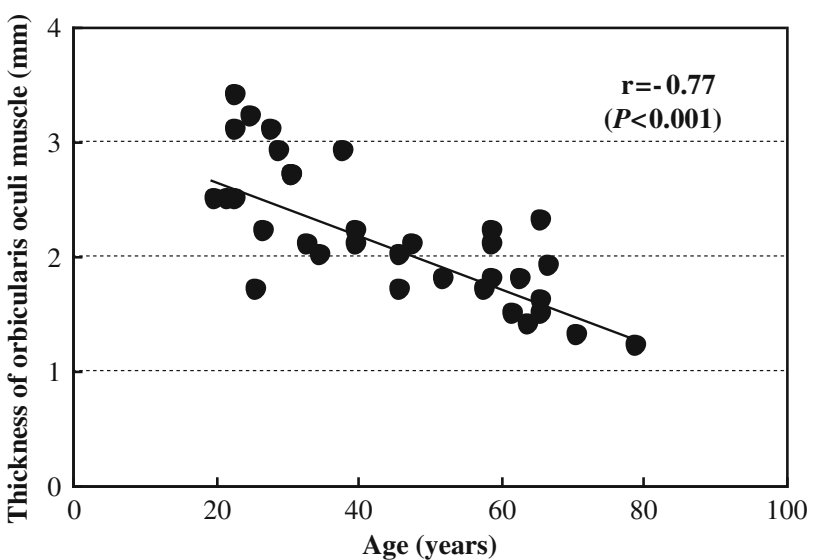

Fig. 3 Correlation between orbicularis oculi muscle thickness and age. A strong and significant negative correlation was found between orbicularis oculi muscle thickness and age $(r=-0.77 ; P<0.001)$. Specifically, with age, the orbicularis oculi muscle becomes thinner

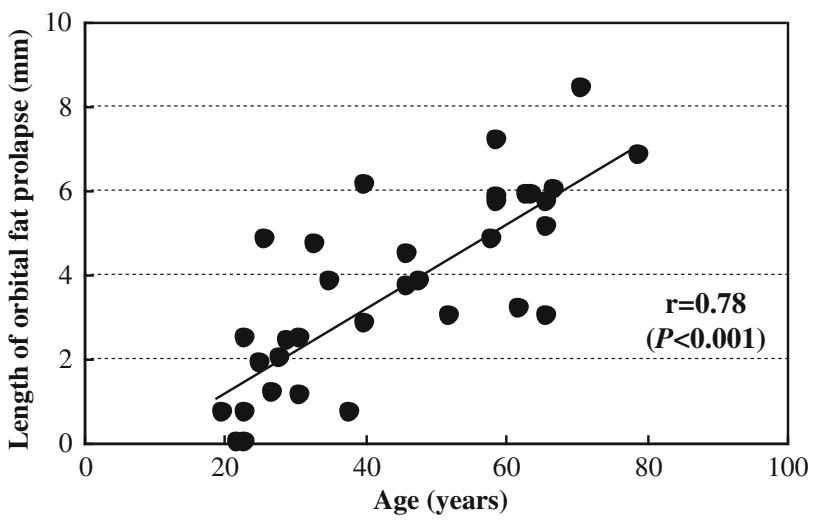

Fig. 4 Correlation between length of orbital fat prolapse and age. A strong and significant positive correlation was found between orbital fat prolapse length and age $(r=0.78 ; P<0.001)$. Specifically, with age, orbital fat prolapse length increases

tear trough depression, loss of skin elasticity, orbicularis prominence, and a triangular malar mound [4]. Trussle and Byrd [9] described the combined major effects of gravity and orbicularis oculi muscle activity on midface aging. Carriquiry et al. [10] and Hamra [11, 12] determined that aging results in the lower orbicularis oculi muscles becoming atonic and ptotic. Furnas [5] reported that baggy eyelids are caused by laxity in the orbicularis oculi. Chen et al. [6] and Darcy et al. [13] analyzed intraorbital fat herniation using CT and magnetic resonance imaging (MRI), respectively, showing the relationship between increased orbital fat prolapse and progressive age.

Gallagher et al. [14] and Janssen et al. [15] reported that skeletal muscle volume decreased with aging. We guessed that this aging change also occurred with the orbicularis oculi muscles. In our study, we focused on the orbicularis

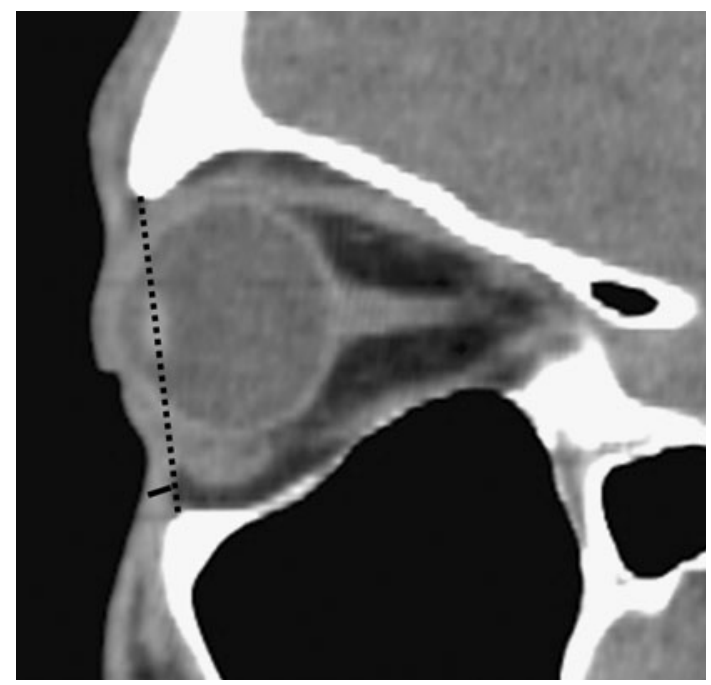

Fig. 5 A 22-year-old man who showed no orbital fat prolapse. The thickness of the orbicularis oculi muscle shown with the black line is $2.5 \mathrm{~mm}$

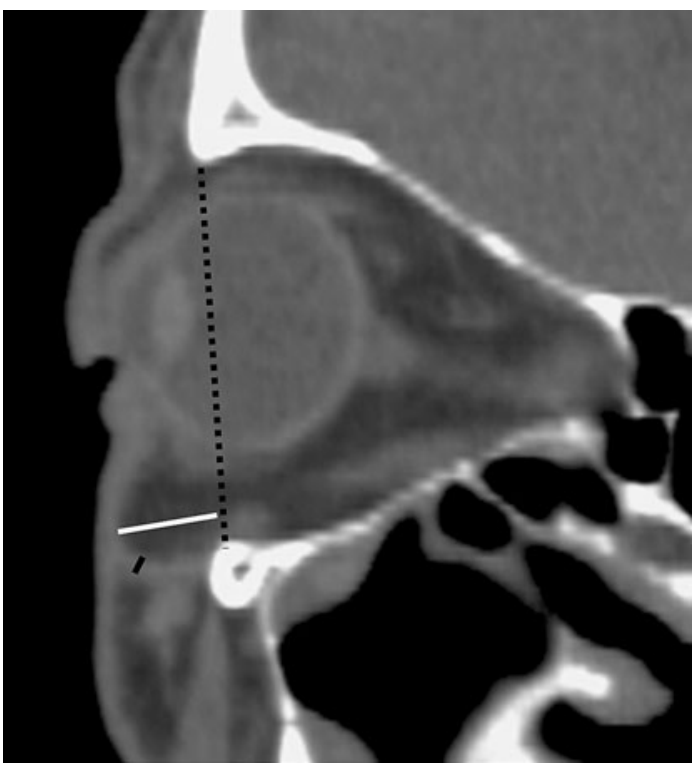

Fig. 6 A 63-year-old man who showed orbital fat prolapse. The orbital prolapse length $(5.9 \mathrm{~mm})$ is shown by the white line. The orbicularis oculi muscle thickness $(1.8 \mathrm{~mm})$ is shown by the black line

oculi muscle as the cause of baggy eyelids and evaluated the relationship between orbicularis oculi muscle thickness, orbital fat prolapse length, and age. The relationship between orbicularis oculi thickness and orbital fat prolapse length were demonstrated by MDCT measurements. Precise reconstructed images of the lower eyelids were created for accurate measurement of orbicularis oculi thickness and orbital fat prolapse length. Sagittal reconstructed images created vertical to the orbit were used for measurements. 
Orbicularis oculi thickness and orbital fat prolapse were significantly correlated $(P=-0.78)$. Specifically, as the orbicularis oculi muscle became thinner, the orbital fat prolapse became more prominent. Age was associated with orbicularis oculi muscle thickness and orbital fat prolapse. Specifically, with younger age, the orbicularis oculi muscle was thicker and the orbital fat prolapse was milder. With increased age, orbicularis oculi thickness was reduced and orbital fat prolapse lengthened. These findings indicate that orbicularis oculi muscle is thinner and orbital fat prolapse longer with age, and these are a major cause of facial aging. Additionally, in our analysis, orbicularis oculi muscle thickening was reduced with aging in both males and females.

In a report released by Okuda et al., the benefit of MDCT is discussed as an objective method for evaluating facial aging [16]. In this study, baggy eyelids were evaluated among age-related changes on the face. The degree of baggy eyelids was quantified by MDCT. The relationship between orbicularis oculi thickness and baggy eyelids, which has been conventionally understood, is supported by the objective evidence presented in this report. In addition, the association between these factors and age also is demonstrated. Because this evaluation method using MDCT is highly objective, imaging features are supplemented with previously established aesthetic plastic surgical knowledge about baggy eyelids, and clinical applications are suggested by objective evaluation using imaging features.

\section{Conclusion}

Imaging with MDCT aided in visualization of lower eyelid structures, and analysis of these structures provided further insight into detailed changes associated with baggy eyelids. This study objectively demonstrated that the main factors leading to baggy eyelids are orbicularis oculi muscle thickness, orbital fat prolapse length, and age. With increased age, orbicularis oculi thickness is reduced and orbital fat prolapse lengthens. Decreased orbicularis oculi muscle thickness leads to orbital fat prolapse.

Open Access This article is distributed under the terms of the Creative Commons Attribution Noncommercial License which permits any noncommercial use, distribution, and reproduction in any medium, provided the original author(s) and source are credited.

\section{References}

1. del Campo AF (2008) Update on minimally invasive face lift technique. Aesthet Surg J 28:51-61

2. Gassner HG, Rafii Young A, Murakami C, Moe KS, Larrabee WF (2008) Surgical anatomy of the face: implications for modern face-lift techniques. Arch Facial Plast Surg 10:9-19

3. Larrabee WF, Makielski KH, Henderson J (2004) Surgical Anatomy of the Face, 2nd edn. Lippincott Williams \& Wilkins, Philadelphia

4. Goldberg RA, McCann JD, Fiaschetti D, Simon GJB (2005) What causes eyelid bags? Analysis of 114 consecutive patients. Plast Reconstr Surg 115:1395-1402

5. Furnas DW (1978) Festoons of orbicularis muscle as a cause of baggy eyelids. Plast Reconstr Surg 61:540-546

6. Chen YS, Tsai TH, Wu ML, Chang KC, Lin TW (2008) Evaluation of age-related intraorbital fat herniation through computed tomography. Plast Reconstr Surg 122:1191-1198

7. Belden CJ, Mancuso AA, Schmalfuss IM (1999) CT features of congenital nasal piriform aperture stenosis: initial experience. Radiology 213:495-501

8. Kahn DM, Shaw RB Jr (2008) Aging of the bony orbit: a threedimensional computed tomographic study. Aesthet Surg J 28: 258-264

9. Trussler AP, Byrd HS (2009) Management of the midface during facial rejuvenation. Semin Plast Surg 23:274-282

10. Carriquiry CE, Seoane OJ, Londinsky M (2006) Orbicularis transposition flap for muscle suspension in lower blepharoplasty. Ann Plast Surg 57:138-141

11. Hamra ST (1992) Composite rhytidectomy. Plast Reconstr Surg 90:1-13

12. Hamra ST (1992) Repositioning the orbicularis oculi muscle in the composite rhytidectomy. Plast Reconstr Surg 90:14-22

13. Darcy SJ, Miller TA, Goldberg RA, Villablanca JP, Demer JL, Rudkin GH (2008) Magnetic resonance imaging characterization of orbital changes with age and associated contributions to lower eyelid prominence. Plast Reconstr Surg 122:921-929 discussion 930-931

14. Gallagher D, Visser M, De Meersman RE, Sepúlveda D, Baumgartner RN, Pierson RN, Harris T, Heymsfield SB (1997) Appendicular skeletal muscle mass: effects of age, gender, and ethnicity. J Appl Physiol 83:229-239

15. Janssen I, Heymsfield SB, Wang ZM, Ross R (2000) Skeletal muscle mass and distribution in 468 men and women aged 18-88 years. J Appl Physiol 89:81-88

16. Burmahl B (2011) 3D CT imaging used to evaluate facial aging. In: Hovsepian DM (ed). RSNA News 21(3):5. http://www.rsna.org/ Publications/rsnanews/March-2011/upload/RSNA_News_March2011. pdf 